\title{
Gesetzesinitiativen zur Pflege heiß diskutiert
}

— Vom 8. bis 10. Juni fand der 19. Hauptstadtkongress Medizin und Gesundheit im Berliner CityCube statt. Die jährliche Leitveranstaltung der Gesundheitsbranche besuchten mehr als 8.000 Teilnehmer. In rund 200 Einzelveranstaltungen diskutierten Spitzenpolitiker, Wissenschaftler und Vertreter aller Berufsgruppen der Gesundheitswirtschaft die Zukunft des Gesundheitswesens.

Das Leitthema hieß in diesem Jahr "Innovationen“. Bereits in der Eröffnungsveranstaltung hatte Annette Widmann-Mauz, MdB, Parlamentarische Staatssekretärin beim Bundesgesundheitsminister, die Haltung der Bundesregierung deutlich gemacht, dass das Solidarsystem nur nützliche Innovationen finanzieren könne. Die Innovationsstrategie der Bundesregierung sehe dafür drei Voraussetzungen: Erstens müssten „echte Innovationen einen wirklichen Fortschritt für Lebensqualität und Lebenschancen" bringen. Zweitens müssten sich
Innovationen am Versorgungsbedarf orientieren. Und drittens dürften Innovationen "keine Eintagsfliegen" sein.

Auch in der Pflege sind Innovationen zunehmend wichtig. So kann der Prototyp eines Roboters Heimbewohnern nicht nur Wasser ausschenken, sondern per Gesichtserkennung auch für jeden Einzelnen die getrunkene Menge registrieren. Zudem stellen sich Fragen, wie: Können Roboter helfen, den Pflegenotstand zu entschärfen? Wie wäre das unter ethischen Kriterien zu beurteilen? Oder: Sind die Telepflege, das vernetzte Heim oder die Dienstplan-App echte Zukunftsfelder?

Im Fokus standen außerdem die zahlreichen Gesetzesinitiativen zur Pflege. Der Patientenbeauftragte der Bundesregierung, Staatssekretär Karl-Josef Laumann, hob die Wichtigkeit der vielfältigen Gesetzesvorhaben der Bundesregierung zur Stärkung der Pflege hervor: „Pflege hat in dieser Legislaturperiode einen ganz zentralen Stellen- wert eingenommen." Kontroverse Diskussionen gab es vor allem beim Pflegeberufegesetz und bei der Personalbemessung, auch die Pflegekammer erhitzte die Gemüter. Doch auch Themen wie die Akademisierung der Pflege im Mix der Gesundheitsberufe, aktuelle Entwicklungen hinsichtlich der Patientensicherheit, der Dauerbrenner Entbürokratisierung sowie die Zukunft der Pflegeheime und der ambulanten Versorgung standen auf dem Programm.

Aufsehen erregte der Auftritt des US amerikanischen Pioniers der regenerativen Medizin, Prof. Dr. Anthony Atala. Der Forscher und sein Team beschäftigen sich am Wakefield Forest Institute mit der künstlichen Erzeugung menschlichen Gewebes und menschlicher Organe aus körpereigenen Zellen. Schon jetzt leben Patienten mit Organen aus Atalas Labor, wie etwa mit einer Harnblase, einer Vagina und Knochensegmenten. Ziel der Wissenschaftler ist es, die Transplantationsmedizin von ihrem Engpass bei Spenderorganen zu befreien.

www.hauptstadtkongress.de

\section{DPR zur Entscheidung des Ärztetags zur Aufgabenverteilung Delegation statt Substitution?}

— "Die Entschließung des 119. Deutschen Ärztetags zur Delegation statt Substitution greift zu kurz", kritisiert Andreas Westerfellhaus, Präsident des Deutschen Pflegerates e.V. (DPR), eine aktuelle Entscheidung des Deutschen Ärztetags. „Wir brauchen eine umfassende Diskussion über die sinnvolle und dringend benötigte Neuverteilung von Aufgaben aller Gesundheitsberufe, insbesondere und gleichermaßen von Ärzten und Pflegefachpersonen".

"Das in Deutschland vorherrschende arztzentrierte Gesundheitssystem gehört auf den Prüfstand gestellt. Wir benötigen ein kompetenzorientiertes System", so Westerfellhaus weiter. Ein Veto bei einzelnen Berufsgruppen dürfe es dabei nicht geben. Denn angesichts der gewaltigen pflegerischen und ärztlichen Aufgaben der nächsten Jahrzehnte müsse es bei der Neuordnung der Aufgaben "um die Leistungsmöglichkeiten und nicht um das Prestige" gehen. Ein "weniger an alten und starren Strukturen" wäre an dieser Stelle

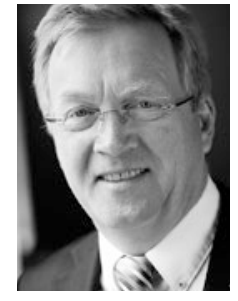

Andreas Westerfellhaus Präsident des Deutschen Pflegerates (DPR)

sehr hilfreich. „Wie die Ärzteschaft, sieht dabei auch der DPR die Versorgungsqualität und damit die Patientensicherheit als oberstes Gebot an. Es ist aber nicht die Ärzteschaft allein, die die Patientensicherheit garantieren kann. Wir fordern dazu auf, die seit langem vorgesehenen Modellvorhaben über die Festlegung ärztlicher Tätigkeiten zur Übertragung auf Berufsangehörige der Alten- und Krankenpflege zur selbstständigen Ausübung von Heilkunde nach $\S 63$ Abs. 3c SGB V endlich umzusetzen", ergänzt der Präsident des DPR.

www.deutscher-pflegerat.de
Alles richtig?

Die korrekten Antworten der PflegeKollegs lauten:

Krebsmedizin heute

$1 a, 2 b, 3 c, 4 c, 5 a, 6 b, 7 a, 8 b, 9 c, 10 c$

Prophylaxen II

$1 c, 2 a, 3 b, 4 a, 5 c, 6 a, 7 b, 8 c, 9 b, 10 a$

Wunddébridement

$1 b, 2 a, 3 a, 4 c, 5 b, 6 c, 7 b, 8 c, 9 b, 10 a$

Vorschau!

Die PflegeKollegs im September:

Stürze vermeiden

Im Team - In Balance - Mit Motivation

Erkrankungen der Leber

Hepatitis - Komplikationen - Ernährung 